> Après avoir suivi brillamment deux ans de cours préparatoires à la formation universitaire qui devait le mener au professorat, Gregor Mendel entre subitement dans un monastère augustin en 1843. A-t-il renoncé à son projet professoral pour devenir prêtre ou a-t-il seulement repoussé ce projet? Afin de déterminer les raisons de son comportement, nous étudions dans cet article ses années de formation secondaire, en complétant et en corrigeant les données d'un curriculum vitae rédigé par lui-même en avril 1850, pour le joindre à une demande d'habilitation à l'enseignement secondaire. <

En 1834, Johann Mendel, fils de paysans silésiens, alors âgé de douze ans, fut envoyé à la ville pour y suivre des études secondaires, comme le rapportent les biographes Hugo Iltis [1] et Vitezslav Orel [2]. À cette époque, cette portion de la Silésie et la Moravie ${ }^{1}$ voisine faisaient partie de l'empire des Habsbourg où les paysans étaient encore soumis à la Corvée ${ }^{2}$. Dans sa classe sociale, peu d'enfants (les garçons seulement) arrivaient à dépasser le niveau de la formation primaire. Après avoir terminé six ans d'éducation secondaire, le jeune Mendel, en suivant deux années supplémentaires d'étude de Philosophie, affirme son projet d'entrer à I'Université. Mais en 1843, après avoir suivi brillamment ces enseignements qui lui donnaient accès aux études supérieures, il entre au monastère augustin Saint-Thomas d'Alt-Brünn (Brno) sous le nom de religion Gregor et s'engage à étudier la théologie pour devenir prêtre. Ce changement d'orientation nous interroge et nous chercherons à découvrir les circonstances qui l'ont alors mené à renoncer aux études universitaires. Comment a-t-il pris la décision d'entrer dans les ordres?

Les informations sur la vie du jeune Mendel sont très rares, car il n'est devenu célèbre que bien des années après sa mort. Toutefois, il la décrit lui-même dans un

${ }^{1}$ Correspondant respectivement à la partie située au sud-ouest de la Pologne et à la partie orientale de la République Tchèque.

${ }^{2}$ Travail non rémunéré imposé par un souverain ou seigneur à ses sujets.

\section{Gregor Mendel fut-il soumis à la corvée avant de devenir moine en 1843 ?}

\section{Christiane Nivet}

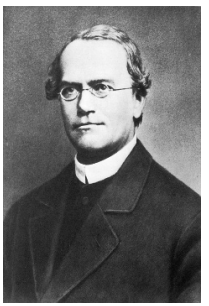

Université Paris7 Denis Diderot, Paris, France. christiane.nivet@univ-paris-diderot.fr

curriculum vitae rédigé en avril 1850, joint à son dossier de demande d'habilitation à l'enseignement secondaire.

Ce document fut publié en allemand par Hugo Iltis en 1926 [3] puis en anglais en 1954 [4]. Ces deux publications s'accompagnent de fac-similés présentant partiellement le texte manuscrit. La traduction française de ce curriculum que nous proposons rend compte à la fois du texte holographe et des deux versions imprimées. Ce texte est un document de circonstance, hâtivement baptisé autobiographie par Iltis, et pris pour tel par tous ceux qui voulaient connaître la vie du fondateur de la génétique.

Nous montrons ici qu'en réalité, le récit que Mendel fait de sa jeunesse néglige certains aspects déterminants de sa vie quotidienne; nous essaierons de la reconstituer en utilisant les données de la lettre de recommandation écrite en 1843 par Friedrich Franz, son professeur de physique [1]. Elle fournit des indications précieuses sur les circonstances de son entrée dans les ordres. De plus, des informations archivées dans les divers établissements qui ont accueilli Mendel sont accessibles : elles font connaître les dates où Mendel s'est inscrit pour suivre différents enseignements, celles de ses absences durant ces enseignements et les notes, toujours excellentes, qu'il a obtenues. Enfin nous ferons intervenir les circonstances du drame familial qui perturba l'avenir du jeune Mendel ${ }^{3}$.

\section{Comment le jeune Mendel fut envoyé dans un établissement d'enseignement secondaire}

D'après ses biographes $[1,2]$, l'intelligence du jeune Johann fut décelée par son instituteur. Le prêtre de sa paroisse, Johann Schreiber, un

\footnotetext{
${ }^{3}$ Dans cet article, nous publions la version française de ce curriculum vitae découpé en extraits en italique
} numérotés, afin de permettre au lecteur d'en recomposer la séquence originale intégrale. 
pédagogue réputé, persuada ses parents de faire suivre des études secondaires à leur unique fils malgré leurs possibilités financières limitées.

À cette époque, en Moravie, le cursus secondaire uniquement littéraire se décomposait en quatre ans de Grammaire, suivis de deux ans d'Humanités; toutes les disciplines étaient enseignées en allemand par le même professeur [6]. Avant d'être admis à l'Université, le candidat effectuait encore deux années d'études de Philosophie dans un Institut approprié.

Voici comment Mendel se présente; il parle de lui à la troisième personne, conformément à l'usage de l'époque :

Le respectueux soussigné est né en l'an 1822 à Heinzendorf, en Silésie, où son père exploitait un petit domaine agricole. Après avoir reçu une instruction élémentaire dans l'école du dit village, puis au Collège des Piaristes à Leipnik, il fut admis en première classe de Grammaire au Gymnasium Impérial et Royal de Troppau en 1834. (CV 1)

Mendel est né dans une famille catholique de langue allemande. Son père, Anton Mendel, aurait voulu que Johann, seul garçon d'une famille de trois enfants, lui succédât un jour. Mais il ne pouvait ignorer que les diplômes étaient pour son fils la seule chance d'échapper à la vie difficile de la classe paysanne d'alors, encore soumise à la Corvée. La classe d'études primaires, quand elle existait pour les garçons, n'était pas ouverte aux filles. Mais Rosine, la mère de Johann, avait appris à lire et à écrire avec un oncle instituteur, lorsqu'il faisait la classe aux garçons du village dans leur maison familiale. Elle fut le parent le plus favorable aux études de son fils. La famille était assez aisée mais endettée à cause des améliorations apportées au domaine par Anton : dans leur village souvent sujet à des incendies, les Mendel furent les premiers à remplacer leur maison de bois par une maison en pierre.

Comme la plupart des petits paysans qui ne vivent pas en milieu urbain, Johann fera ses études secondaires comme pensionnaire au Gymnasium ${ }^{4}$ le plus proche de la ferme familiale, dans la ville de Troppau (de nos jours Opava) en zone germanophone, à une quarantaine de kilomètres du domicile familial.

\section{Début des études secondaires : Grammaire (1834-1838)}

D'après Hugo Iltis [1], dès le début de sa scolarité à Troppau, l'enfant n'a pas une vie matérielle facile: faute de numéraire, les parents de Mendel n'ont inscrit leur fils que pour le coucher et la demi-pension, se réservant de lui procurer un complément de nourriture aussi souvent que possible. Du jeune Mendel, le biographe dit que : «c'est un garçon robuste, de petite taille, large d'épaules, qui dut bien souvent souffrir de la faim pendant sa scolarité et qui, pendant ses vacances d'été, ne se reposait pas vraiment car il aidait aux champs et à l'étable chez ses parents ».

Après avoir mentionné dans son curriculum vitae son entrée au Gymnasium de Troppau, Mendel évoque sans les décrire ses quatre premières années d'études au cours desquelles il a poursuivi une scolarité brillante et apparemment sans histoire.

\footnotetext{
${ }^{4}$ Établissement d'enseignement secondaire équivalent au lycée.
}

Quatre ans plus tard, une succession de malheurs mit ses parents hors d'état d'assumer les dépenses exigées par la poursuite de ses études, et il arriva donc que le soussigné, alors âgé d'à peine seize ans, se retrouva dans la triste situation de pourvoir seul à sa subsistance. C'est pourquoi il suivit les cours destinés aux «Candidats à l'enseignement dans les écoles primaires et au professorat privé » dispensés par l'École Normale du district de Troppau. Ses résultats à l'examen lui ayant valu un certificat d'aptitude avec la meilleure mention, il réussit, comme répétiteur privé, à gagner de quoi vivre chichement durant le cours de ses Humanités. (CV 2)

Donc, à cette époque, à la suite de quatre ans d'études brillantes, Mendel a confirmé haut la main son aptitude à suivre des études. De quelle nature est la succession de malheurs qui se sont produits vers 1838 ? Quel rôle la famille de Mendel a-t-elle joué au cours de ses études? Dans son curriculum vitae, document officiel censé montrer qu'il avait été surtout un élève exemplaire (non un fils dévoué), Mendel pouvait-il décrire pleinement les conséquences, qu'il dut assumer, de cette situation familiale?

\section{Qu'advint-il exactement en 1838 ?}

La «succession de malheurs » juste évoquée par Mendel dans son curriculum vitae, comporte, outre de mauvaises récoltes successives, l'accident corporel grave de son père, renversé par la chute d'un arbre durant son service de Corvée. Iltis nous dit qu'Anton Mendel devait travailler pour le seigneur des lieux trois jours par semaine: un jour et demi avec ses deux chevaux et le reste manuellement. Il a été blessé par un arbre coupé, ce qui suppose la coupe et le transport d'arbres la moitié du temps.

L'incapacité physique d'Anton Mendel eut pour conséquence immédiate pour son fils l'arrêt de la prise en charge des frais exigés par sa demi-pension et son hébergement; sans doute aussi, une réduction de la nourriture fournie par la famille. En même temps, le jeune homme est soumis à une forte pression psychologique: son père invalide attend de son fils qu'il reprenne la ferme et qu'il assiste sa mère et ses deux sœurs. Mais, au lieu d'interrompre ses études, confirmant ainsi sa résolution de poursuivre son cursus scolaire, Johann passe au plus vite un examen pour devenir répétiteur, afin de subvenir à ses besoins.

\section{Fin des études secondaires (1838-1840)}

A-t-il vraiment abandonné sa famille dans la détresse? Dans de telles circonstances, a-t-il cessé son assis- 
tance pendant les vacances scolaires? Ne l'aurait-il pas, au contraire, augmentée, prenant du temps sur ses études pour suppléer à l'invalidité de son père? C'est ce qu'il fit, puisque, l'année suivante (183940), sous prétexte de maladie, Mendel écourta sa scolarité de deux mois: délaissant le Gymnasium à la Pentecôte (début juin), il n'y revint qu'à la rentrée d'octobre.

Ses biographes moraves $[1,2]$ considèrent que la maladie explique les interruptions de sa scolarité, mais ils ne tiennent pas compte de la situation dramatique où se trouvait sa famille. Ses absences ne se produisant jamais pendant le repos hivernal agricole, mais en juin et juillet, alors que le travail agreste bat son plein, nous suggérons plutôt que loin d'être malade et alité, Mendel a, la plupart du temps, manqué la classe pour participer aux travaux de la ferme.

Dans sa demande d'habilitation et pour éviter de perdre sa crédibilité d'excellent élève et de futur enseignant, il n'avouera pas avoir privilégié parfois sa famille au détriment de ses études. À partir de 1838, la vie du jeune homme se déroule donc ainsi : il poursuit brillamment ses études secondaires à plein temps, enseigne pour assumer maigrement sa subsistance et travaille à la ferme dès qu'il le peut, non seulement pendant ses vacances scolaires, mais encore à l'occasion d'absences du Gymnasium dites «pour maladie ». C'est dans ces difficiles conditions qu'il termine avec brio sa scolarité secondaire en 1840 .

\section{Une autre époque dramatique (1840-1841)}

Les conditions déjà difficiles devinrent extrêmement pénibles au cours de ses années d'études pré-universitaires. Revenons au curriculum vitae pour connaître la suite d'un récit qui nous paraît quelque peu édulcoré.

En 1840, quand il quitta le Gymnasium avec son diplôme de fin d'études secondaires, son premier souci fut de se procurer les moyens nécessaires à la poursuite de ses études. Il fit pour cela des tentatives répétées en offrant ses services comme précepteur à Olmütz (Olomouc), mais ses efforts restèrent vains, faute de relations et de recommandations. Le chagrin de voir ses espoirs déçus et la perspective angoissante et triste que lui offrait l'avenir furent ressentis de façon si violente qu'il tomba malade et qu'il dut, pour se remettre, passer une année chez ses parents. (CV 3)

II n'existait pas à Troppau d'établissement assurant les deux années d'études pré-universitaires, dites de Philosophie ; il dut donc se rendre à Olomouc, autrefois capitale tchèque de la Moravie. Mendel prétend que c'est par manque de relations qu'il ne trouve pas de poste de précepteur dans une ville inconnue, étrangère. Désespéré, il rentre chez ses parents, et passe avec eux une année à se rétablir.

Peut-on croire que, pendant si longtemps, le chagrin ait désarmé ce jeune homme réputé pour la fermeté de son caractère et de sa constitution physique [1] ? Pouvons-nous croire qu'étant donné la situation de sa famille, il lui a fallu un an à la ferme pour guérir un chagrin? II faut donc tirer de ce roman une explication.

D'après IItis [1], depuis I'hiver 1838 et l'accident d'Anton Mendel, la situation de la famille est dramatique. Au cours des mois qui suivirent l'accident, la santé du père, loin de s'améliorer, se dégrada tellement qu'il devint inapte au travail, laissant la ferme sans ouvrier et ses devoirs de corvéable à l'abandon, au grand dam de son maître.

Mais, contrairement à ce qu'il dit, Johann Mendel s'est inscrit à Olomouc à l'automne 1840 en trouvant sur place de quoi subsister pendant la première année de ses études de Philosophie. II avait donc assisté aux cours du premier semestre et avait commencé les examens de semestre avec succès [6] lorsqu'il dut quitter les épreuves précipitamment, sans doute à la suite d'une aggravation subite de l'état de son père. II passa le deuxième semestre de l'année à la ferme et ne reprit ses études qu'à l'occasion de la cession du domaine familial à un autre paysan.

En effet, Veronica, la fille aînée de la famille Mendel, épousa Alois Sturm et Anton Mendel lui céda sa ferme par contrat en août 1841. Dans ce contrat, la propriété était évaluée, une pension devait être payée aux parents et diverses sommes allouées aux enfants. La cession de la ferme à son beau-frère, Alois, procurait à Johann le strict minimum pour continuer ses études et exigeait de Sturm le paiement des frais de la première messe au cas où Johann deviendrait prêtre. Elle ménageait aussi son éventuel retour à la ferme dans le cas contraire [7].

Cet acte enregistre le fait qu'Anton Mendel abandonne définitivement l'espoir de voir son fils lui succéder et constitue le premier document où la possibilité d'une éventuelle entrée en religion de Johann est évoquée.

Relayé en principe à la ferme grâce à cette alliance avec un homme valide, Johann reprit ses études, son maigre pécule se trouvant augmenté d'une partie de la dot de sa sœur Theresia, sa cadette de sept ans. Compte tenu de l'âge de sa sœur, cet emprunt se fit grâce à l'intercession de Rosine, leur mère, qui se porta probablement garant de son éventuel remboursement, confirmant par là son soutien, en toutes circonstances, aux ambitions professorales de son fils. Mendel conserva pour sa sœur une reconnaissance dont il s'acquitta honorablement en assumant les frais d'études des trois fils de celle-ci. Mendel a caché son départ de la session d'examen car il aurait pu compter pour un échec : il semblerait qu'il n'existe qu'une seule excuse acceptée pour manquement à un parcours scolaire qui se doit d'être impeccable : la maladie!

\section{Études de Philosophie (1841-1843)}

Comme un curriculum vitae n'est pas le lieu où faire part de ses problèmes familiaux ni de son dénuement, Mendel écrit, sans plus de commentaire, que tout s'arrange à la rentrée de 1841-1842 : 
L'année suivante, le respectueux soussigné se trouva enfin dans la situation espérée de poursuivre ses études à Olmütz en couvrant ses besoins les plus indispensables grâce à des leçons particulières. En mobilisant toutes ses forces, il réussit à mener à bien les deux années de philosophie. (CV 4)

Johann Mendel effectua donc ses études de Philosophie avec des moyens matériels toujours aussi limités et s'absenta encore longuement pour cause de maladie vers la fin du deuxième semestre de chacune de ces années, ce qui incite à penser qu'il a probablement continué à aider son beau-frère à la ferme autant qu'il le pouvait : ce dernier, lui aussi probablement redevable de la Corvée, avait en charge ses sœurs, sa mère et les frais de la maison spécialisée dans laquelle Anton Mendel avait dû se retirer en janvier 1842. C'est ce que nous retenons de la phrase: « $\varepsilon$ n mobilisant toutes ses forces, il réussit à mener à bien les deux années de Philosophie».

$\varepsilon$ n absence d'informations sur la nature précise des maladies avancées comme responsables de ses absences au cours de ses études de philosophie, Hugo Iltis [1] insinue que la cause en aurait été « des troubles névrotiques » consécutifs à des «crises psychologiques dues à l'adolescence ». Quant à Orel [2], il attribue à des «désordres nerveux particuliers » les congés «pour maladie » que Mendel a dû prendre « aux moments critiques de sa vie».

Au contraire, nous présentons ici des arguments qui montrent que les arrêts de sa scolarité furent mis à profit par Mendel pour assumer, lui aussi, le service de Corvée. L'astreinte à la Corvée qui portait sur la famille étant héréditaire et les rapports entre seigneurs et paysans sans merci, il nous paraît possible que le seigneur des lieux (dont on ne sait pas grand-chose si ce n'est l'histoire fictive rapportée par Iltis d'une comtesse qui aurait enseigné les sciences naturelles aux enfants du village [8]) ait exigé de Johann qu'il réponde de son statut héréditaire de corvéable : après 1650 , le paysan tchèque doit 150 jours de corvée par an et sa condition semble très dure à tous les observateurs étrangers [9], et, devant l'absence du père, on a pu exiger du jeune Mendel, alors âgé de seize ans, qu'il exécute le travail de Corvée assumé par son père auparavant. Ce qui signifie que pendant les quatre premières années de Gymnasium, l'enfant aurait bénéficié d'une dispense car, lors de ces années, aucune absence n'est enregistrée [6].

Lorsque Mendel écrit «qu'il sentit qu'il ne lui serait pas possible de soutenir plus longtemps de tels efforts», il ne pense pas au travail requis par les leçons qu'il donnait aux enfants de bourgeois qui allaient au Gymnasium. Mais c'est bien de la Corvée dont le caractère épuisant vient probablement non seulement du type de travail mais aussi et surtout du fait qu'à cette période de l'année (mi-mai à fin septembre), le jeune homme exécutait probablement ce travail hebdomadaire à temps complet et non pas à mi-temps, comme son père faisait tout au long de l'année.

Enfin, si on cherche à identifier le type d'activité à laquelle Mendel pouvait avoir contribué, on peut ajouter l'entretien d'un four à chaux, spécialité de son village [1], ce qui suppose le transport de combustibles et de pierres calcaires puis l'entretien du feu à 1000 degrés pour obtenir de la chaux vive.
On peut conclure que les maladies invoquées n'ont été que des subterfuges destinés à cacher le cumul de la Corvée avec les études du jeune Mendel. L'attestation de bonne santé exigée pour son admission au monastère, délivrée par le Docteur Schwarz le 7 septembre 1843, vient confirmer nos hypothèses.

Mendel cache donc son astreinte à la Corvée qui aurait pu être considérée comme incompatible à la poursuite d'études de qualité. Cette information aurait-elle suffi à le disqualifier comme futur professeur de Gymnasium ? L'entrée au monastère était à l'époque l'unique possibilité pour lui de quitter son statut et les efforts dont parle Mendel ne correspondent pas au fait de cumuler études et préceptorat, comme ses biographes ont l'air de le penser.

\section{Comment Mendel se résigna à devenir moine}

L'origine de la décision de Mendel d'entrer au monastère augustin Saint-Thomas d'Alt-Brünn (Brno) est énoncée clairement dans le curriculum vitae tel qu'il a été publié $[3,4]$. Voici ce qui est dit de la période qui précède son entrée au couvent:

Mais le respectueux soussigné sentit qu'il ne lui serait pas possible de soutenir plus longtemps de tels efforts. Après avoir terminé ses études de Philosophie, il se vit contraint de s'engager dans un état qui l'exemptât de l'amer souci de trouver des moyens de subsistance. Ses conditions de vie décidèrent du choix de ce nouvel état. Il sollicita et obtint, en l'an 1843, d'être admis au monastère des Augustins Saint-Thomas d'Alt-Brünn. (CV5)

Mendel dit être entré dans les ordres en 1843, sans vocation particulière, pour des raisons matérielles qui cachent son besoin de changer de statut et se délivrer de la Corvée. Arrivé aux portes de l'Université à 21 ans, Mendel déclare que : «ses conditions de vie décidèrent du choix de ce nouvel état ». Peut-on mettre en doute la réalité de son épuisement physique et mental ? On entrevoit mieux maintenant comment fut prise sa décision d'entrer à Saint-Thomas : à bout de force, épuisé par neuf ans de lutte pour s'instruire, gagner sa vie, assister sa famille en déroute et plus particulièrement, pendant les cinq dernières années assumer ses obligations en rapport avec la Corvée.

II parle de cette transition sans enthousiasme et laisse entendre que ce choix de vie ne l'attire pas particulièrement. Néanmoins, comme nous allons le voir, il en espère une contrepartie, car il n'abandonne pas son projet d'aller à l'Université pour pouvoir se présenter à l'examen d'habilitation et devenir - enfin - enseignant titulaire de l'enseignement secondaire. 


\section{Pourquoi Mendel fut-il accepté au monastère Saint-Thomas en 1843 ?}

En juin 1843, le Supérieur du monastère augustin Saint-Thomas, Franz Cyril Napp, cherchait de nouveaux impétrants dont certains, par leur mérite, seraient susceptibles de devenir professeurs d'histoire naturelle, car son établissement était censé fournir une partie des enseignants de l'Institut de Philosophie et du Collège de Théologie de la ville de Brno [1,2]. Dans ce but, il avait écrit, au début de juin, à son collègue et ami Friedrich Franz, professeur de physique à l'Institut de Philosophie d'Olomouc, pour lui demander de lui signaler les élèves dont les qualités en feraient de bons candidats à l'entrée au monastère de Brno et dont l'envoi à l'Université ne coûterait au monastère que les frais de trois semestres universitaires.

À la mi-juillet, ayant trouvé deux candidats, Friedrich Franz n'en recommande qu'un seul : Johann Mendel, «à cause de ses excellents résultats au cours des deux années de Philosophie, presque le meilleur dans ma spécialité... et de sa force de caractère » [1]. Il précise que, sa connaissance du tchèque étant insuffisante, Mendel est néanmoins tout disposé à en acquérir la maîtrise pendant ses années de formation théologique. Il fut ainsi accepté à Saint-Thomas sans avoir à se présenter à l'habituel entretien préalable à toute candidature. Un mois séparant la demande de Napp de la réponse de Franz, on constate que c'est le délai dont Mendel disposa pour se décider à postuler, et s'il en accueillit sans enthousiasme la proposition, ce fut néanmoins avec un soulagement probable qu'il accepta de demander à être reçu au monastère.

Grâce à cette démarche sa situation matérielle changea alors totalement. Le bien-être physique, si favorable aux études quelles qu'elles soient, rendit au soussigné son courage et son énergie et c'est avec beaucoup de plaisir et d'amour qu'il étudia les matières classiques au programme de son année de probation. (CV6)

Mendel entre donc à Saint-Thomas dans une certaine euphorie.

Il consacrait ses loisirs aux petites collections de minéralogie et de botanique que le monastère mettait à sa disposition. Plus il se familiarisait avec les sciences naturelles, plus s'approfondissait le goût qu'il leur portait. Bien qu'il ne pût bénéficier dans cette étude d'aucun soutien oral, alors que dans cette discipline plus peut être que dans toute autre science, la méthode autodidactique est extrêmement ardue et ne mène que lentement au but, il se passionna si fort à partir de ce moment-là pour l'étude de la nature qu'il n'épargna aucun effort pour combler ses lacunes grâce à ses propres recherches et aux avis de personnes d'expérience. En 1846, il suivit également les cours d'agriculture, de pomiculture et aenologie de l'Institut de Philosophie de Brno. (CV7)

Dès son intégration dans le monastère, on constate que Mendel découvre et se passionne pour les sciences naturelles. Napp lui permet de s'inscrire aux divers cours d'agriculture, professés par son ami et collaborateur Franz Diebl, que Mendel suivit avec un grand intérêt et pour lesquels il obtint d'excellentes notes.

Après avoir achevé ses études de théologie en 1848, le respectueux soussigné reçut de son supérieur l'autorisation de préparer le
«Rigorosum» de Philosophie (doctorat en philosophie). L'année suivante, au moment où il allait se présenter à cet examen, on lui demanda d'accepter le poste de professeur remplaçant au Gymnase Impérial et Royal de Znaïm et il accéda à cette demande avec plaisir. (CV8)

Mendel s'abstient de mentionner ce qui pourrait rappeler un autre épisode turbulent de sa vie : il omet la fonction de curé qu'il a occupée à Brno du 22 juillet 1848 au début octobre 1849, à cause de laquelle il a engendré avec d'autres moines une pétition acerbe $[5](\rightarrow)$.

$(\rightarrow)$ Voir le Forum de C. Nivet, $m / s^{\circ} 11$, novembre 2004, page 1050

Sans doute grâce à l'entregent de l'abbé Napp et dans le désordre qui a suivi la période révolutionnaire, le ministre viennois Lazansky nomme Mendel professeur suppléant à Znaïm le 28 septembre 1849. On observe que l'abbé en informe l'évêque le 4 octobre (au lieu de le consulter) et que Mendel se rend immédiatement à Znaïm le 7 octobre [5]. Cette nomination a un caractère d'urgence qui s'explique par une mesure non préparée d'allongement de deux ans du cursus scolaire des Gymnasia [14]. Ce qui créa une panique chez les fonctionnaires chargés de trouver le personnel enseignant: ils engagèrent transitoirement même ceux qui, n'ayant pas suivi les études universitaires exigées pour enseigner en secondaire, avaient néanmoins suivi les études de philosophie : tel fut le cas de Mendel. On remarque cependant que sa tentative de passer l'habilitation à la fin de cette année d'enseignement fut un peu présomptueuse.

Dès ses débuts en tant que professeur remplaçant, il s'attacha tout particulièrement à présenter à ses élèves de la façon la plus aisément compréhensible les matières qui lui étaient confiées. Et il est en droit de penser que cet effort n'est pas demeuré vain, car les quatre années durant lesquelles il avait gagné sa vie comme enseignant privé lui avaient permis d'accumuler une expérience suffisante concernant les résultats que peuvent obtenir les élèves et leurs différents niveaux de capacité intellectuelle. (CV9) Mendel essaie de valoriser son expérience passée de maître privé.

Le respectueux soussigné pense avoir ainsi présenté un résumé de l'histoire de sa vie. Sa triste jeunesse lui a enseigné précocement le sérieux de l'existence et lui a aussi appris à travailler. Alors même qu'il jouissait des fruits de la sécurité économique, il garda vivant en lui le désir d'être autorisé à gagner sa vie. Le respectueux soussigné serait heureux de satisfaire aux attentes de l'illustre Commission des 
examinateurs et de voir ainsi ses vœux exaucés. Il n’épargnera assurément aucun effort ni aucun sacrifice pour s'acquitter très ponctuellement de ses devoirs.

Znaïm, le 17 avril 1850.

Gregor Mendel Professeur remplaçant au gymnase Imperial et Royal de Znaïm

(CV10)

Dans la dernière partie de ce curriculum vitae, Mendel précise qu'il demande à être habilité à l'enseignement secondaire afin de gagner sa vie. Cela était possible pour les moines de St Thomas. Formés aux frais du monastère, ils enseignaient ensuite dans les institutions pédagogiques de Brno et étaient rétribués en retour par les instances locales. On trouve ici le souci de Mendel d'assurer la vie matérielle de ses neveux pendant leur éducation en retour du don que sa sœur cadette lui avait fait jadis de son héritage.

\section{Conclusion}

Nous avons vu que le curriculum vitae rédigé par Mendel donne une version bien édulcorée de la vie ardue qu'il a menée avant d'entrer à Saint Thomas. Ses biographes moraves l'ont acceptée sans prendre en considération la solidarité de Mendel avec sa famille, ni l'éventualité de son astreinte à la Corvée, pas plus que les omissions commises afin de ménager ses chances d'être habilité comme professeur. De confession catholique sans vocation religieuse particulière, il devient moine pour des raisons strictement matérielles qui cachent son souhait probablement inavouable de quitter son statut de paysan corvéable.

Prenant en compte les circonstances que nous venons de décrire, il est plus facile de comprendre la violence de sa réaction fin juillet 1848 $[2,5,10]$, à l'annonce imprévue de sa nomination à vie pour le travail pastoral à la fin de ses études de théologie. Alors que Mendel avait atteint pour la deuxième fois le seuil de l'Université, après tant d'années d'épreuves et de sacrifice, il s'est encore retrouvé dans la position de celui qui ne décide pas et se voit simplement passé d'une sujétion à une autre. Il est désespéré encore une fois de ne pas pouvoir réaliser sa vocation de professeur pour laquelle, à la fin de ses études secondaires, il avait poursuivi en vain trois ans éprouvants d'études supérieures de philosophie cumulées avec les impératifs de la Corvée. Sous l'emprise de cette énorme déception, et dans l'ambiance enthousiaste de la révolution, il est probablement, comme nous l'avons suggéré [11], à l'origine de la violente pétition du 8 août 1848 où grondent amertume et agressivité contre l'institution monacale mais aussi contre le pouvoir politique impérial. Cette pétition a été lue publiquement devant l'assemblée Constituante de Kremsier fin janvier 1849 alors que le pouvoir impérial était en voie de restauration $[10,13](\rightarrow)$.

$(\rightarrow)$ Voir le Forum de C. Nivet, $m / s^{\circ} 4$, avril 2006, page 430

On peut s'interroger sur le fait que, Iltis, premier

biographe de Mendel, ne mentionne pas l'existence de cette pétition qui n’est révélée que par Orel qui, néanmoins, évite de parler des conséquences qui en ont résulté pour Mendel.

Après que l'abbé Napp a révoqué Mendel en tant que curé, sa nomination comme professeur suppléant par un ministre viennois [14] signifie-t-elle que Mendel est, alors, à nouveau sur le chemin désiré ?

\section{SUMMARY}

Was Gregor Mendel subjected to chores before becoming a monk in 1843 ?

Our knowledge of the young Mendel's life prior to his admission to the monastery comes essentially from the curriculum vitae submitted in 1850 . His first biographer Hugo Iltis used this document as a sort of autobiography, although the document contained various voluntary omissions and inaccuracies. We have sought the reasons for these and in so doing have discovered why Mendel's entry into religion had become ineluctable. $\diamond$

\section{RéFÉRENCES}

1. Iltis H. Life of Mendel. New York: Norton, $1932: 336$ p. Life of Mendel, $2^{e}$ ed New York: Hafner, 1966. Traduction anglaise de Gregor Johann Mendel Leben, Werk und Wirkung. Berlin : Springer, 1924.

2. Orel V. Gregor Mendel: the first geneticist. New York: Oxford University Press, 1995.

3. Iltis H. Gregor Mendels Selbstbiographie. Genetica (The Hague) 1926, 8 : 329-34.

4. Iltis A. Gregor Mendel's autobiography. J Heredity 1954 ; 45 : 231-4. Traduction française réalisée par Hélène Arnold et Daniel Blanchard.

5. Nivet C. Une maladie énigmatique dans la vie de Gregor Mendel. Med Sci (Paris) $2004 ; 20: 1050-3$.

6. Weiling F. Historical study: Johann Gregor Mendel, 1822-1884. Am J Med Genet $1991 ; 40: 1-25$.

7. Orel V, Armogathe JR. Mendel, un inconnu célèbre. Paris : Belin, 1985.

8. Klein J, Klein N. Solitude of a humble genius: Gregor Johann Mendel, vol. 1. Formative years. New York: Springer, 2013

9. Schuster PM. Moving the stars, Christian Doppler, his life, his works and principle (chap. 5). New York : Living Editions, 2005.

10. Nivet C. 1848 : Gregor Mendel, le moine qui voulait être citoyen. Med Sci (Paris) $2006 ; 22: 430-3$

11. Bérenger J. Lexique historique de l'Europe danubienne $X V l^{e}-x X^{e}$ siècle. Paris : Armand Colin, 1976.

12. Krizenecky J. Gregor Johann Mendel 1822-1884. Text und Quellen zu seinem Wirken und Leben. Leipzig : Ambrosius Barth Verlag, 1965.

13. Bérenger J. L'Autriche-Hongrie 1815-1918. Paris : Armand Colin, 1994

14. Cohen GB. Education and middle-class society in Imperial Austria 18481918. New York: Purdue University Press, 1996.

\section{TIRÉS À PART}

C. Nivet
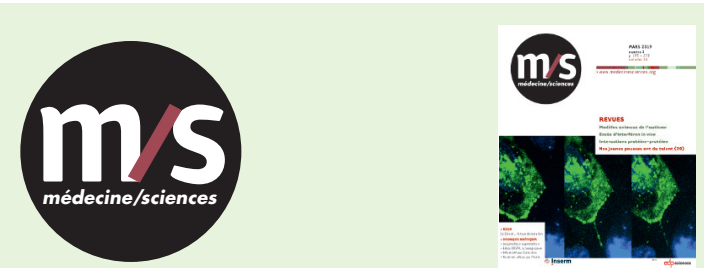

Abonnez-vous à médecine/sciences

Bulletin d'abonnement page 90 dans ce numéro de $\mathrm{m} / \mathrm{s}$ 